\title{
Role of matrix metalloproteinases in radiation-induced lung injury in alveolar epithelial cells of Bama minipigs
}

\author{
HAIYING YUE ${ }^{1}, \mathrm{KAI} \mathrm{HU}^{1}$, WENQI LIU ${ }^{1}$, JIE JIANG $^{1}$, YUHUA CHEN ${ }^{2}$ and RENSHENG WANG ${ }^{1}$ \\ ${ }^{1}$ Department of Radiotherapy, The First Affiliated Hospital of Guangxi Medical University, Nanning, Guangxi 530021; \\ ${ }^{2}$ Department of Pathology, Guangxi Province Maternity and Child Care Hospital, Nanning, Guangxi 530002, P.R. China
}

Received November 13, 2014; Accepted July 7, 2015

DOI: $10.3892 /$ etm.2015.2658

\begin{abstract}
Radiation-induced lung injury (RILI) is a common complication associated with thoracic radiotherapy. The aim of the present study was to investigate the effects of a single 15-Gy dose of right-thoracic lung irradiation on the expression levels of matrix metalloproteinases (MMPs) and other proteins in the alveolar epithelial type II (AE2) cells of Bama minipigs. All minipigs received either right-thoracic irradiation or sham irradiation under anesthesia, and were sacrificed at 4, 8, 12 or 24 weeks after irradiation. Collagen deposition was measured using Masson's trichrome staining. Surfactant protein A (SP-A), transforming growth factor $\beta 1$ (TGF $\beta 1$ ), MMP2, MMP9, vimentin and E-cadherin protein expression levels were evaluated using western blot analysis, and the MMP2 and MMP9 gelatinase activities were tested using gelatin zymography. SP-A and $\alpha$-smooth muscle actin ( $\alpha$-SMA) co-localization was visualized using double immunofluorescence staining. At each time-point following irradiation, a significant increase in TGF $\beta 1, \alpha$-SMA, MMP2, MMP9 and vimentin protein expression levels and MMP2 and MMP9 gelatinase activity were observed in the irradiated lungs compared with the sham-irradiated controls. By contrast, SP-A and E-cadherin protein expression levels decreased in a time-dependent manner post-irradiation. SP-A and $\alpha$-SMA co-localization was observed in irradiated alveolar epithelial cells. These data demonstrate that E-cadherin, SP-A, MMP2 and MMP9 may function as sensitive predictors of RILI. Epithelial-mesenchymal transition (EMT) occurs in the irradiated lungs of Bama minipigs, and MMP2 and MMP9 may contribute to EMT in AE2 cells by regulating TGF $\beta 1$.
\end{abstract}

Correspondence to: $\mathrm{Dr}$ Rensheng Wang, Department of Radiotherapy, The First Affiliated Hospital of Guangxi Medical University, 6 Shuangyong Road, Nanning, Guangxi 530021, P.R. China

E-mail: 13807806008@163.com

Key words: minipigs, radiation-induced lung injury, epithelial-mesenchymal transition, matrix metalloproteinase-2, matrix metalloproteinase-9
Therefore, EMT may serve a crucial function in the development of RILI.

\section{Introduction}

Radiotherapy is a crucial therapy for the treatment of thorax-associated neoplasms. However, during a course of treatment with radiotherapy, the lung tissue is inevitably damaged by the radiation dose, which is above the biologically tolerable threshold. Radiation-induced lung injury (RILI) is a common complication of radiotherapy and a key dose-limiting factor, which may reduce the probability of tumor control and affect the patient's quality of life (1). The pathology of RILI is complex, and includes radiation-induced pneumonitis and fibrosis (2). Although intensive study in the previous decades has elucidated a number of the associated underlying mechanisms, involving, for example, cells and cytokines, the specific mechanism remains unclear (3).

The alveolar wall extracellular matrix (ECM) consists of a basement membrane and an interstitial matrix. It acts as a physical barrier to the passage of macromolecules and cells, provides physical tissue support and anchorage sites for cells, and serves as a substrate for cell migration and a medium for diffusible cell signaling molecules. Matrix metalloproteinases (MMPs) are a large family of related proteolytic enzymes that specifically degrade ECM components. MMP2 and MMP9, also known as gelatinases, are key enzymes in basement membrane degradation.

The alveolar epithelium is composed of type I and II cells, which are morphologically and functionally distinct. Alveolar epithelial type I (AE1) cells are the first type to be affected and to undergo apoptosis when injury occurs. Alveolar epithelial type II (AE2) cells synthesize and secrete pulmonary surfactant, and also proliferate and transdifferentiate into AE1 cells, which play an important role in maintaining the structure and function of the alveoli. AE2 cell injury has been associated with the development of lung fibrosis (4), and AE2 cells from patients with idiopathic pulmonary fibrosis and other experimental pulmonary fibrosis diseases express high levels of epithelial-mesenchymal transition (EMT)-associated protein markers $(4,5)$. The process of EMT results in a loss of epithelial polarity, cytoskeletal reorganization, downregulation of epithelial marker expression (e.g., E-cadherin), the acquisition of mesenchymal markers [e.g., $\alpha$-smooth muscle 
actin ( $\alpha$-SMA) and vimentin], and an increase in cell invasiveness and migration (6). A recent study observed that pro-surfactant protein-c (pro-SP-c) and $\alpha$-SMA are co-localized in the irradiated lung alveoli of $\mathrm{FVB} / \mathrm{N}$ mice, suggesting that the AE2 cells achieved a mesenchymal phenotype (7). However, the anatomical structure of the rodent lung differs considerably from that of humans. Furthermore, the radiation response in the lung varies with species and strain (8). For Bama minipigs, the single-dose radiation dose-effect curve is comparable to that in humans, as is the anatomical structure and physiology of the lung (9). Therefore, the present study aimed to determine whether AE2 cells are able to transdifferentiate into a mesenchymal-like phenotype by dynamically observing the protein expression levels of surfactant protein $\mathrm{A}$ (SP-A), E-cadherin, $\alpha$-SMA and MMPs. In addition, the present studied aimed to elucidate the possible role of MMP2 and MMP9 in RILI using Bama minipigs as experimental subjects.

\section{Materials and methods}

Minipigs and collection of lung specimens. This study was approved by the Committee on the Ethics of Animal Experiments of Guangxi Medical University (Permit No. SCXKGUI 2009-0002; Nanning, China). All surgery was performed under sodium pentobarbital anesthesia, and all animals were treated humanely.

Male Bama minipigs were supplied by the Experimental Animal Center of Guangxi Medical University. Forty minipigs, aged 2-3 months and weighing $10-15 \mathrm{~kg}$, were randomly divided into control and irradiated groups ( $\mathrm{n}=20$ per group). Following the intraperitoneal administration of anesthesia, using $3 \%$ sodium pentobarbital $(30 \mathrm{mg} / \mathrm{kg})$, minipigs in the irradiated group received a single 15-Gy dose of right-thoracic ${ }^{60} \mathrm{Co}$ gamma irradiation (field, $9 \times 10 \mathrm{~cm}$; source-surface distance, $80 \mathrm{~cm}$; absorbed dose, $3 \mathrm{~Gy} / \mathrm{min}$ ), using a GWXJ80 ${ }^{60} \mathrm{Co}$ radiotherapy treatment unit (Nuclear Power Institute of China, Chengdu, China). The minipigs in the control group received sham irradiation. Five minipigs in each group were randomly sacrificed at 4, 8, 12 and 24 weeks post-irradiation, respectively. Subsequently, the thorax was opened and right lung tissue was resected under anesthesia. The upper lobe was fixed in $10 \%$ neutral formalin for histological and immunohistochemical analysis, and the lower lobe was snap frozen in liquid nitrogen and stored at $-80^{\circ} \mathrm{C}$ until use.

Histopathology and immunohistochemistry. Lung sections (4 $\mu \mathrm{m})$ were subjected to hematoxylin and eosin (H\&E) and Masson's trichrome staining. The sections were deparaffinization and antigen retrieval was conducted using a high pressure method with sections on ice in $0.01 \mathrm{M}$ citrate bufer. Next, sections were incubated with mouse monoclonal $\alpha$-SMA antibodies (1:400; ab8211 Abcam, Cambridge, England) at $4^{\circ} \mathrm{C}$ overnight, and then were assayed using a streptavidin-peroxidase kit (Beyotime Institute of Biotechnology, Haimen, China), according to the manufacturer's instructions. Positive cells displayed a brownish-yellow cytoplasm, indicating that they were smooth muscle cells or activated myofibroblasts that synthesize collagen and certain components of the ECM.
Double immunofluorescence staining. Double-color immunofluorescence analysis was used to identify $\alpha$-SMA and SP-A expression in the lung AE2 cells. Briefly, following deparaffinization and antigen retrieval, sections were blocked using normal donkey serum (Molecular Probes Life Technologies, Carlsbad, CA, USA) for $20 \mathrm{~min}$ at $37^{\circ} \mathrm{C}$, then incubated with a mixture of anti- $\alpha$-SMA (1:100, Abcam) and anti-SP-A (1:100, Abbiotec, San Diego, CA, USA) antibodies at $4{ }^{\circ} \mathrm{C}$ overnight. Nuclei were counterstained with 4'-6-diamidino-2-phenylindole (Beyotime Institute of Biotechnology) for $8 \mathrm{~min}$, and the sections analyzed using a Nikon A1 laser scanning confocal microscope (Nikon Corporation, Tokyo, Japan).

Western blot analysis. Lung tissue (40-80 mg) was rinsed, shredded and homogenized in $600 \mu 1$ radioimmunoprecipitation lysis buffer with $1 \mathrm{mM}$ phenylmethylsulfonyl fluoride (PMSF), and the supernatant was quantified using a bicinchoninic acid protein assay kit (Beyotime Institute of Biotechnology). Following 10\% SDS-polyacrylamide gel electrophoresis with $40 \mu \mathrm{g}$ sample per lane, the proteins were transferred to a polyvinylidene difluoride (PVDF) film (EMD Millipore, Billerica, MA, USA). The film was then blocked with $1 \mathrm{X}$ Tris-buffered saline with Tween 20 (TBST) for $1 \mathrm{~h}$ at room temperature, and incubated with primary antibodies against GAPDH (1:5,000; ABS16), MMP2 (1:500; MAB3308), MMP9 (1:2,000; AB6001; Millipore), TGF $\beta 1$ (1:50; 5559-100; BioVision, Inc., Milpitas, CA, USA), E-cadherin (1:500), vimentin (1:1,000; ab8069; Abcam) and SP-A (1:200; Abbiotec) at $4^{\circ} \mathrm{C}$ overnight. After washing with phosphate-buffered saline, the sections were incubated with donkey anti-mouse Alexa Fluor 594-conjugated (A21202) and anti-rabbit Alexa Fluor 488-conjugated secondary antibodies (A21206; 1:5,000; Molecular Probes Life Technologies) at room temperature for $1 \mathrm{~h}$. After washing with $1 \mathrm{X}$ TBST, the film was incubated with anti-mouse or anti-rabbit IgG horseradish peroxidase-labeled antibodies (Asbio Technology, Inc., Guangzhou, China) at room temperature for $1 \mathrm{~h}$. Finally, the film was visualized using enhanced chemiluminescence according to the manufacturer's instructions (ECL+; Pierce Biotechnology, Inc., Rockford, IL, USA) and scanned using an ImageQuant LAS500 imaging system (GE Healthcare Life Sciences, Chalfont, UK). Scanned films were analyzed semiquantitatively for the densitomateic signal of bands on the western blot using software included with the ImageQuant LAS500.

Gelatin zymography. Lung tissue $(50 \mathrm{mg}$ ) was rinsed with normal saline and shredded using operating scissors, then homogenized in $600 \mu \mathrm{l}$ lysis buffer $(150 \mathrm{mM} \mathrm{NaCl}, 10 \mathrm{mM}$ $\mathrm{CaCl}_{2}, 10 \mathrm{~g} / \mathrm{l}$ Triton X-100 and $1 \mathrm{mM}$ PMSF). Protein concentration was quantified using an identical protocol to that of the western blot analysis. Following $8 \%$ SDS-polyacrylamide gel electrophoresis with $40 \mu \mathrm{g}$ sample per lane, the gel was stained according to the manufacturer's instructions using an MMP zymography assay kit (Applygen Technologies, Inc., Beijing, China). The gel was then scanned using an Odyssey two-color infrared scanning laser imaging system and analyzed using Odyssey software, version 2.1 (LI-COR Biosciences, Lincoln, NE, USA). 

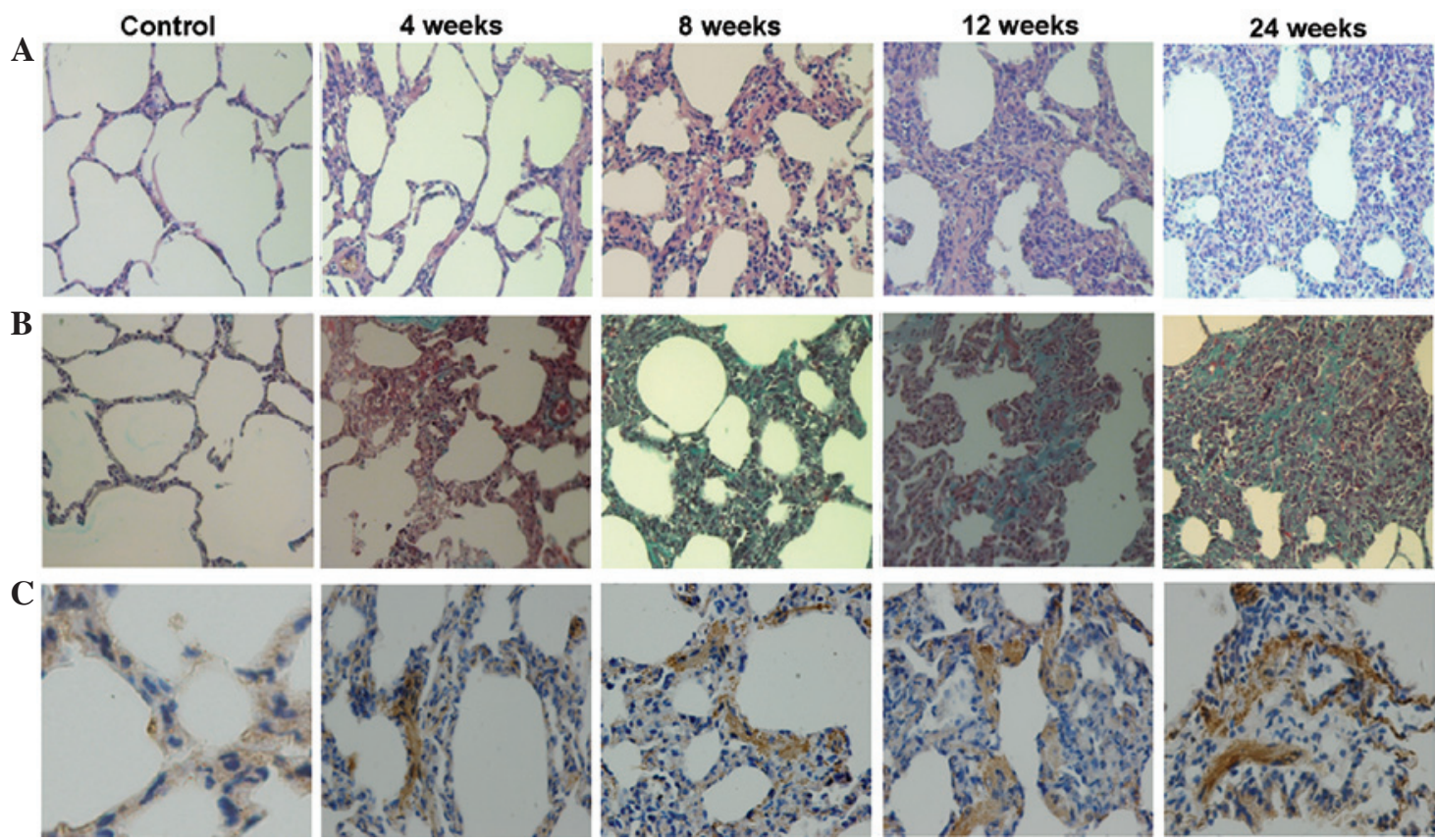

Figure 1. Irradiating the lungs of Bama minipigs lead to lung fibrosis, collagen deposition and increased inflammatory cell infiltration. Minipigs received right-thoracic irradiation with a single 15-Gy dose. Lung tissue sections obtained at weeks 4, 8, 12 and 24 weeks after irradiation were stained using hematoxylin and eosin (H\&E), Masson's trichrome and for $\alpha$-smooth muscle actin ( $\alpha$-SMA). Fields show representative lung images of (A) H\&E staining, (B) Masson's trichrome staining and (C) $\alpha$-SMA staining (magnification, $\mathrm{x} 40$ ). Cytoplasm that exhibited $\alpha$-SMA expression was stained brown.
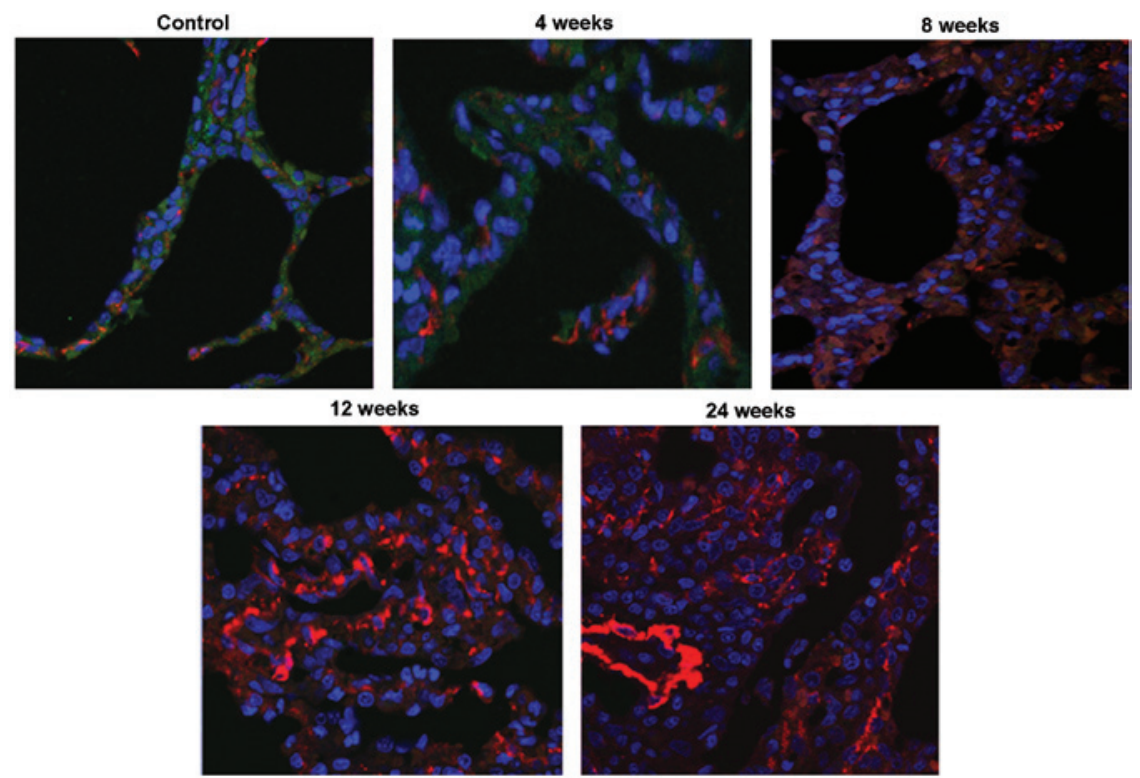

Figure 2. Surfactant protein A (SP-A) and $\alpha$-smooth muscle actin ( $\alpha$-SMA) co-localize in the lungs of Bama minipigs following irradiation. Lung sections $(4 \mu \mathrm{m})$ from control and irradiated minipigs were co-stained with SP-A and $\alpha$-SMA antibodies. In the representative oil microscopy photomicrographs, the SP-A protein is green, $\alpha$-SMA protein is red and nuclei are blue (magnification, $\mathrm{x} 20$ ). Orange results from the overlap of red and green.

Statistical analysis. SPSS software, version 13.0 (SPSS, Inc., Chicago, IL, USA) was used for data processing, and data are expressed as the mean \pm standard deviation. The homogeneity of the variance was tested for the measurement data, and one-way analysis of variance was used for group comparisons. A least significant difference test was used for two groups with variance homogeneity, whereas a Games-Howell test was used for two groups with variance heterogeneity. $\mathrm{P}<0.05$ was considered to indicate a statistically significant difference.

\section{Results}

Histological changes in lung tissue. In the control lung tissue, the structure was clear, the alveolar cavity maintained its integrity, and there was no inflammatory cell infiltration. By contrast, 4 weeks after irradiation, the irradiated lung tissue showed pulmonary interstitial and capillary hyperemia, and diffuse accumulation of inflammatory cells. At 8 and 12 weeks after irradiation, thickened alveolar walls, narrowed alveolar 

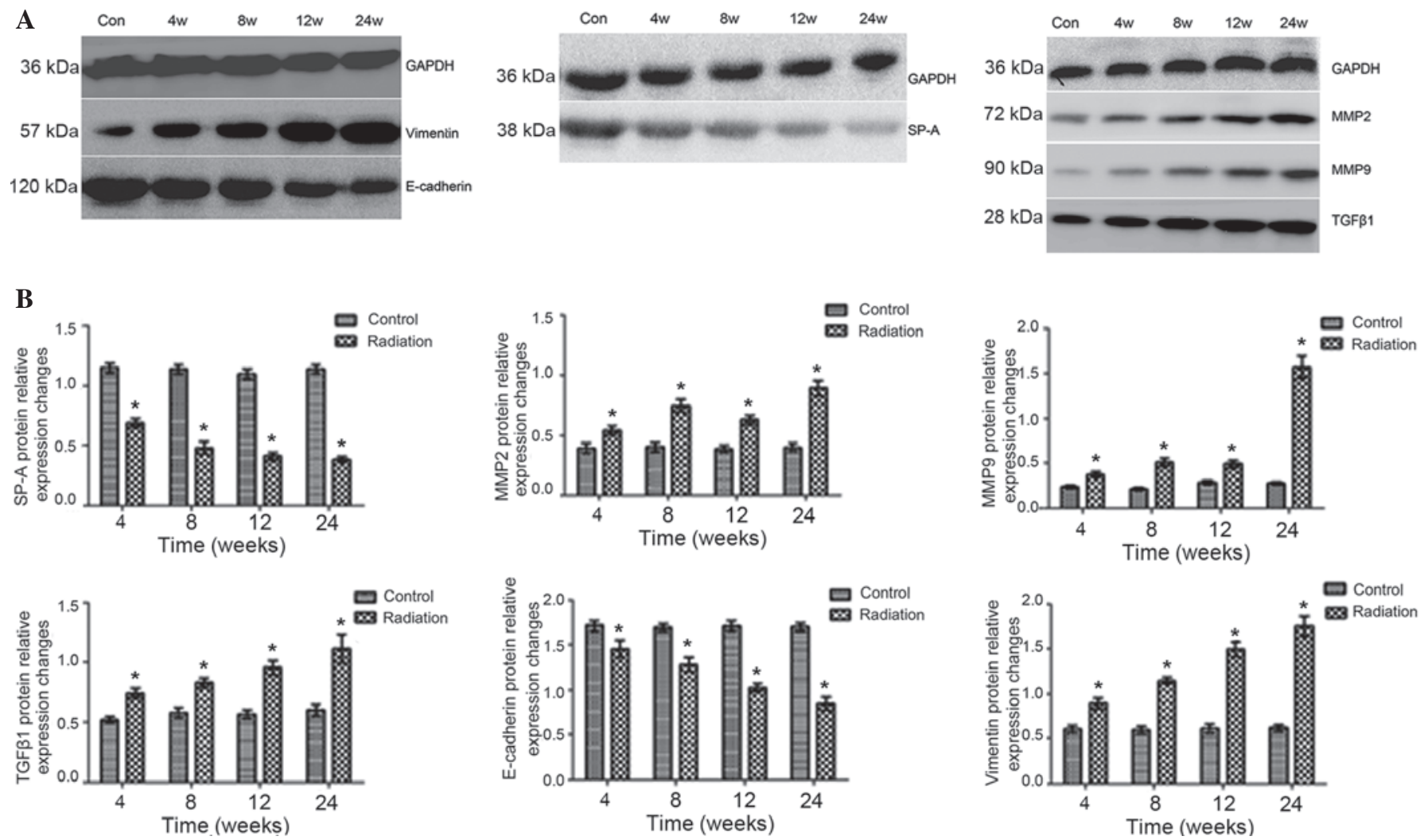

Figure 3. Irradiation differentially modulates SP-A, MMP2, MMP9, TGF $\beta 1$, E-cadherin and vimentin protein expression in the lung. SP-A and E-cadherin protein expression in lung tissue is reduced in a time-dependent manner after irradiation, while irradiation significantly increased TGF $\beta 1$, vimentin, MMP2 and MMP9 protein expression in lung tissue. (A) Representative western blotting results. (B) Densitometry was used to quantify the protein in each lane. All values were normalized against GAPDH values obtained on the same gel, with the exception of SP-A due to its molecular weight. *P<0.01 vs. control. Data are presented as the mean \pm standard deviation. Con, control; w, week; MMP, matrix metalloproteinase; TGF $\beta 1$, transforming growth factor $\beta 1$; SP-A, surfactant protein A.

spaces, an increase in the number of fibroblasts compared with that in the non-irradiated tissue, and fibrous tissue hyperplasia were observed. Inflammatory cells were also observed in the irradiated lung tissue. At 24 weeks after irradiation, multiple fibrotic lesions were present in the irradiated lungs (Fig. 1A). Masson's trichrome staining indicated increasing quantities of collagen fiber deposition in the irradiated lung tissues over time (Fig. 1B). Immunohistochemical staining for $\alpha$-SMA, a marker of smooth muscle cells/myofibroblasts, showed an increased accumulation of myofibroblasts in the irradiated lungs (Fig. 1C).

Co-localization of SP-A and $\alpha-S M A$ protein in the alveoli. A previous study identified pro-SP-c and $\alpha$-SMA protein co-localization in the lungs of FVB/N mice following irradiation, suggesting that EMT may occur in rodents with radiation lung fibrosis (7). Therefore, the right lung tissue of the Bama minipigs was stained for SP-A and $\alpha$-SMA protein following irradiation. The two proteins were observed to be co-localized in the alveoli in the irradiated lungs, and this co-localization was most evident at week 8 post-irradiation. Co-localization of SP-A and $\alpha$-SMA was not observed in the control lung alveoli (Fig. 2), suggesting that radiation caused the trans-differentiation of AE2 cells to a mesenchymal-like phenotype. protein expression in lung tissue. SP-A protein expression levels were reduced and TGF $\beta 1$ protein expression levels were increased following irradiation in a time-dependent manner.

E-cadherin, a calcium-dependent adhesion molecule, mediates the adhesion and tight junctions between epithelial cells. E-cadherin protein expression levels in the irradiated group were reduced in a time-dependent manner following irradiation. However, the protein expression levels of vimentin, a mesenchymal marker, were markedly increased following the radiation treatment.

At each time-point after irradiation, pairwise comparisons revealed that differences between the irradiation and control groups were statistically significant (SP-A and TGF $\beta 1, \mathrm{P}<0.01$; E-cadherin and vimentin, $\mathrm{P}<0.05)$.

Irradiation significantly increases MMP2 and MMP9 protein expression levels. MMP9 protein expression was only moderately increased at 12 weeks post-irradiation, but increased markedly at 24 weeks (week 24 vs. weeks 4, 8 and $12 ; \mathrm{P}<0.01$ ). For MMP2, there was no significant difference in protein expression between weeks 4 and 12 ( $\mathrm{P}>0.05)$, and pairwise comparisons revealed statistically significant differences between the groups at all time-points $(\mathrm{P}<0.01$; Fig. 3).

MMP2 and MMP9 gelatinase activity in lung tissue. After irradiation, increased gelatinolytic activity was observed in the lung tissue at each time-point compared with that in the control lung tissue, and quantitative analysis demonstrated the 
A

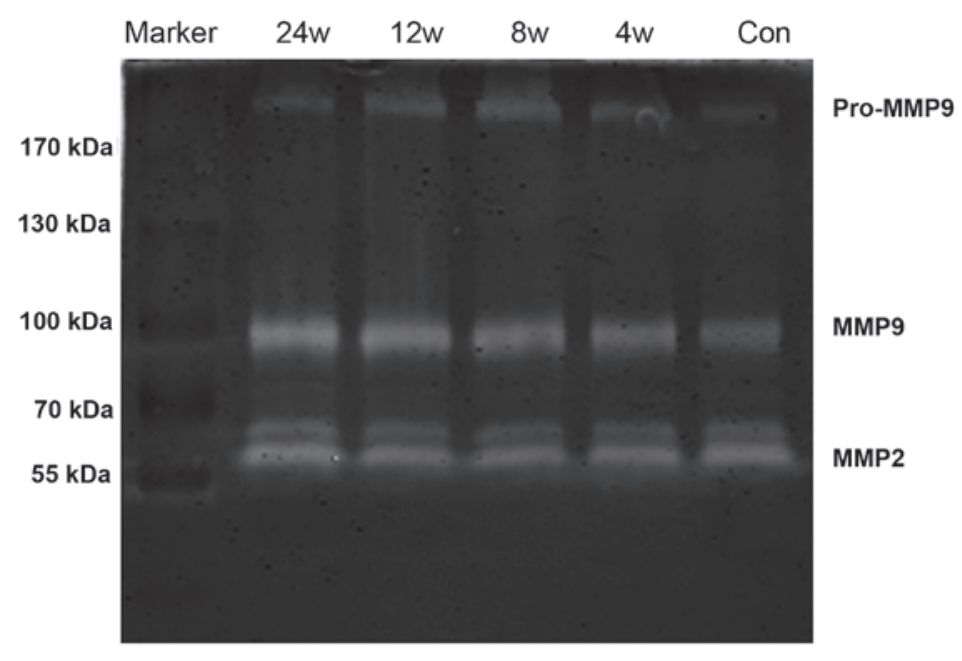

B
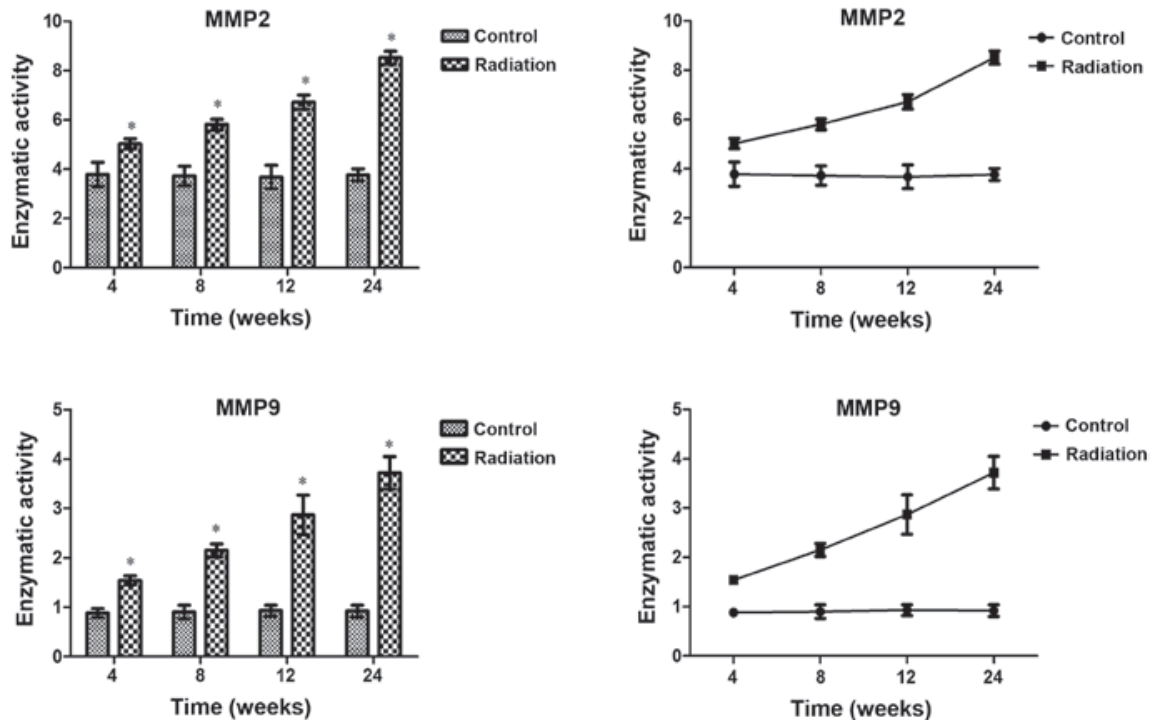

Figure 4. Irradiation leads to a significant increase in gelatinolytic activity in lung tissue. (A) Representative zymogram. (B) Gelatinase activity as determined by densitometric analysis of the zymograms. "P<0.01 vs. control. Data are presented as the mean \pm standard deviation. MMP, matrix metalloproteinase; w, week; Con, control.

time-dependence of the increase in gelatinase activity (Fig. 4). At each time-point after irradiation, pairwise comparisons revealed that differences between the irradiation and control groups were statistically significant (MMP2, P<0.01; MMP9, $\mathrm{P}<0.05)$.

\section{Discussion}

Currently, rodents are typically used to establish animal models of RILI $(10,11)$. However, the sensitivity of the mouse lung to RILI is strain-dependent (8), and minipigs do not exhibit this strain-specific sensitivity (9). The primary pathological features of radiation pneumonitis, such as interstitial edema, capillary hyperemia, and extensive inflammatory cell infiltration, usually appear at 1-3 months after irradiation. Lung fibrosis may occur at 6-12 months after irradiation, and is characterized by excessive fibroblast proliferation and massive collagen deposition (12). In the present study, radiation pneumonitis was observed at 4 weeks (1 month) post-irradiation in minipigs after receiving a single 15-Gy dose of irradiation to the right thorax. At 8 weeks, thickening of the alveolar walls, fibroblasts and inflammatory cells were observed. At 24 weeks (6 months), multiple fibrotic lesions were observed in the irradiated lungs (Fig. 1). These results indicate that an animal model of RILI had been successfully established, and that radiation pneumonitis and fibrosis were not completely separate, but that there was a certain degree of overlap at 8 weeks after irradiation.

Alveolar cells are crucially involved in the maintenance of lung homeostasis. AE1 cells, a terminally differentiated type of cell, are susceptible to injury; however, AE2 cells, are able to proliferate and differentiate, and are considered to be the stem cells of the alveolar epithelia (13). Following damage to the alveolar epithelium, AE2 cells are able to self-renew and transdifferentiate into AE1 cells in order to maintain the integrity of the alveolar epithelium. SP-A, a water-soluble surfactant protein, is secreted by AE2 and Clara cells, and is a key innate immune molecule in the lung. Alcorn and Wright (14) observed that SP-A inhibited inflammatory cytokine production through multiple mechanisms and alleviated lung injury. The transcription regulator nuclear factor- $\kappa \mathrm{B}$ is activated by SP-A, and directly regulates MMP9 expression 
and indirectly induces MMP2 transcription via the transmembrane activation of p53 protein (15). Therefore, SP-A may cause an imbalance the inflammatory/anti-inflammatory medium if inflammatory cytokines in the lung are increased, and affect the protease/antiprotease balance favoring an abnormal breakdown of ECM components. Previous studies on RILI have indicated that SP-A levels are decreased in bronchoalveolar lavage fluid (16), but increased in serum (17), a difference that may be associated with lung injury. In the present study, SP-A protein expression in lung tissue was reduced in a time-dependent manner (Fig. 3), which suggests that the changes in AE2 cells induced by irradiation lead to a reduction in the secretion of surfactants, and promote inflammatory cytokine production, thus influencing MMP2 and MMP9 expression. This situation favors the development of lung injury. In addition, the results of the present study show that the SP-A protein expression level in lung tissue is markedly reduced at week 4 after irradiation, which suggests that SP-A may be used as a predictor of RILI.

A key event in fibrosis is the activation of fibroblasts and their conversion into myofibroblasts, which is characterized by $\alpha$-SMA expression, cytokine production, and crucially, a increased capacity to produce ECM components (18). The origins of myofibroblasts may be divided into three categories: Recruitment and differentiation of resident cells, bone marrow-derived 'fibrocytes' and EMT. A study has demonstrated that myofibroblasts may additionally originate from resident epithelial cells that undergo EMT (19). An in vitro study indicated that the irradiation of RLE-6TN cells induced their transition from an epithelial to a mesenchymal phenotype, which was mediated by the ROS/ERK/GSK-3 $\beta /$ Snail pathway (20). Similarly, $\alpha$-SMA and pro-SP-c co-expression has been detected in the AE2 cells of FVB/N mice following thoracic radiation (7). In the present study, $\alpha$-SMA protein expression levels were significantly increased after irradiation (Fig. 1C). A significant reduction in the expression of the epithelial cell marker E-cadherin and a concomitant increase in the expression of vimentin, a mesenchymal marker, were detected after irradiation (Fig. 3). In addition, double immunofluorescence staining identified $\alpha$-SMA and SP-A protein co-localization in irradiated lung alveoli, which was highest at week 8 after irradiation (Fig. 2), suggesting that AE2 cells had obtained a mesenchymal phenotype. These data indicate that an AE2-to-mesenchymal transition may occur in the irradiated lungs of minipigs and that EMT is a mechanism involved in RILI.

TGF $\beta 1$ is released locally from damaged parenchymal cells and inflammatory cells, and is central to the generation of myofibroblasts and EMT (21). Following activation, myofibroblasts themselves begin to secrete TGF $\beta 1$ and are thereby able to sustain their own activation through a self-stimulatory mechanism, which facilitates the auto-perpetuating process characteristic of fibrosis. In addition, TGF $\beta 1$ is a potent stimulus for the production of ECM components, such as collagen (22), and elevation of TGF $\beta 1$ levels in the plasma of patients during the 4th week of radiotherapy is significantly predictive of RILI (23). In the present study, the continuous increase in the TGF $\beta 1$ protein expression in the irradiated lungs of minipigs further indicates the key function served by TGF $\beta 1$ in the pathogenesis of RILI.
MMPs that degrade ECM components may paradoxically promote collagen deposition. Following lung injury, MMP2 and MMP9 are released from damaged epithelial cells, inflammatory cells or activated myofibroblasts; they degrade and remodel the ECM, and promote cellular migration and activate cytokines such as TGF $\beta 1$, TNF- $\alpha$ and IL-1 $\beta(24,25)$. TGF $\beta 1$ is a key profibrogenic cytokine, which has been implicated as a primary fibrosis trigger in various tissues (26). This suggests that MMP2 and MMP9 are able to facilitate EMT, and previous reports have shown that the two proteins are able to induce EMT in renal tubular epithelial cells $(27,28)$. However, it is unclear whether MMP2 and MMP9 induce EMT in alveolar epithelial cells. Recent evidence has suggested that radiation upregulates MMP expression and activity in various tissues (29-32). The present study indicates that thoracic irradiation induces a marked increase in MMP2 and MMP9 protein expression and gelatinase activity. Furthermore, baseline MMP9 expression and gelatinase activity were only moderately elevated at week 12; however, there was a significant increase at week 24 after irradiation (Figs. 3 and 4). These results indicate that the MMP9-mediated method is more complex compared that of with MMP2, and suggest that MMP9 may play a greater role than MMP2 in RILI. The reduction of SP-A expression induced by irradiation may have caused the MMP9 level to be relatively low at week 12 after irradiation, and TGF $\beta 1$ may also be involved in the inhibition or reduction of MMP9 activity. This may explain why the increase of MMP9 protein expression and gelatinase activity differs from that of MMP2. A previous study indicated that TGF $\beta 1$ is able to downregulate MMP9 gelatinase activity (32), and MMP9 also facilitates the release of TGF $\beta 1$ (25). However, the present study showed that MMP9 protein expression and activity peaked at week 24 after irradiation and TGF $\beta 1$ levels continually increased (Figs. 3 and 4). Further studies may be required to determine the influence of TGF $\beta 1$ on MMP9, and the interaction between the two proteins.

The ECM contains gelatin and collagen, and provides a dynamic support structure on which epithelial cells may grow. In addition, it may influence cellular behavior, including migration, proliferation and morphology (33). Furthermore, ECM is a key source of cytokines, and exerts a typical pleiotropic effect. Although the primary inducer of EMT is TGF $\beta 1$, the significance of the ECM is becoming increasingly apparent, with previous studies suggesting that these components (e.g., type I collagen) influence and augment the profibrotic effects of TGF $\beta 1$ (4,34). For example, studies in various cell types have shown that type I collagen induces EMT $(34,35)$. A previous study indicates that basement membrane architecture is critical in maintaining an epithelial phenotype and that alterations in its composition may promote phenotypic changes (35). Therefore, the interaction between the epithelium and the matrix facilitates the TGF $\beta 1$-induced production of EMT $(4,34)$. Buckley et al $(36)$ observed that type I collagen significantly amplified the effect of TGF 31 -induced EMT in A549 cells treated with TGF $\beta 1$. Additionally, ECM components may have an effect on the activity of fibroblasts (37). Myofibroblasts derived from EMT have been shown to produce ECM components, such as type I collagen (36), indicating that myofibroblasts affect TGF $\beta 1$-induced EMT. TGF $\beta 1$ is an effective stimulus that promotes ECM synthesis (19), 
upregulates MMP2 expression (38) and downregulates MMP9 expression. MMP9, in combination with CD44, is able to stimulate TGF $\beta 1$ production (26), while MMP2 and MMP9 are able to degrade the ECM. This forms a positive feedback loop and continuously promotes the development of RILI, and may result in pulmonary fibrosis. This process a key factor in the consistent development of irradiation-induced lung fibrosis following irradiation. Furthermore, TGF $\beta 1$ is hypothesized to cause fibrosis, and multiple signaling pathways are involved in irradiation-induced lung fibrosis. Thus, further studies are required to clearly determine the mechanisms underlying irradiation-induced lung fibrosis.

On the basis of the present results, we propose a mechanism for RILI in irradiated Bama minipigs. This mechanism involves a complicated interaction among SP-A, TGF $\beta 1$, EMT and ECM which favors the development of RILI, and the participation of MMPs in the development of RILI by interaction with EMT molecules, as described above. Radiation causes a reduction in E-cadherin protein expression levels, an increase in vimentin protein expression and co-localization of $\alpha$-SMA and SP-A in alveolar epithelia, suggesting that EMT occurs in RILI, and that MMP2 and MMP9 serve crucial functions in EMT by regulating TGF $\beta 1$. However, there were certain limitations to the present experiments. For example, SP-A was selected as a marker of AE2 and SP-A was observed to decrease following irradiation, but increased AE2-to-mesenchymal transition was observed at week 8 post-irradiation. However, in the present study, a reduction in the expression level and non-specificity of SP-A possibly led to reduced EMT of AE2 cells at week 12 post-irradiation. However, on the basis of the present data, and potentially with the support of additional future studies to identify the effect of tissue inhibitors of MMPs and types I and IV collagen in RILI may lead to novel preventive interventions or improved treatments for thoracic tumor patients undergoing radiotherapy.

\section{Acknowledgements}

This study was supported by Guangxi Science and Technology Key Projects Fund (no. 1140003A-24).

\section{References}

1. Stone HB, Coleman CN, Anscher MS and McBride WH: Effects of radiation on normal tissue: Consequences and mechanisms. Lancet Oncol 4: 529-536, 2003.

2. Cappuccini F, Eldh T, Bruder D, Gereke M, Jastrow H, Schulze-Osthoff K, Fischer U, Köhler D, Stuschke M and Jendrossek V: New insights into the molecular pathology of radiation-induced pneumopathy. Radiother Oncol 101: 86-92, 2011.

3. Trott KR, Herrmann T and Kasper M: Target cells in raditation pneumopathy. Int J Radiat Oncol Biol Phys 58: 463-469, 2004.

4. Kim KK, Kugler MC, Wolters PJ, Robillard L, Galvez MG, Brumwell AN, Sheppard D and Chapman HA: Alveolar epithelial cell mesenchymal transition develops in vivo during pulmonary fibrosis and is regulated by the extracellular matrix. Proc Natl Acad Sci USA 103: 13180-13185, 2006.

5. Willis BC, Liebler JM, Luby-Phelps K, Nicholson AG, Crandall ED, du Bois RM and Borok Z: Induction of epithelial-mesenchymal transition in alveolar epithelial cells by transforming growth factor- $\beta 1$ : Potential role in idiopathic pulmonary fibrosis. Am J Pathol 166: 1321-1332, 2005.

6. Kalluri R and Weinberg RA: The basics of epithelial-mesenchymal transition. J Clin Invest 119: 1420-1428, 2009.
7. Almeida C, Nagarajan D, Tian J, Leal SW, Wheeler K, Munley M, Blackstock $\mathrm{W}$ and Zhao $\mathrm{W}$ : The role of alveolar epithelium in radiation-induced lung injury. PLoS One 8: e53628, 2013.

8. Williams JP, Brown SL, Georges GE, Hauer-Jensen M, Hill RP, Huser AK, Kirsch DG, Macvittie TJ, Mason KA, Medhora MM, et al: Animal models for medical countermeasures to radiation exposure. Radiat Res 173: 557-578, 2010.

9. Hopewell JW, Rezvani M and Moustafa HF: The pig as a model for the study of radiation effects on the lung. Int J Radiat Biol 76: 447-452, 2000

10. Li M, Abdollahi A, Gröne HJ, Lipson KE, Belka C and Huber PE: Late treatment with imatinib mesylate ameliorates radiation-induced lung fibrosis in a mouse model. Radiat Oncol 4: 66, 2009.

11. Molthen RC, Wu Q, Fish BL, Moulder JE, Jacobs ER and Medhora MM: Mitigation of radiation induced pulmonary vascular injury by delayed treatment with captopril. Respirology 17: 1261-1268, 2012.

12. Travis EL: The sequence of histological changes in mouse lungs after single doses of $\mathrm{x}$-rays. Int J Radiat Oncol Biol Phys 6: 345-347, 1980.

13. Uhal BD: Cell cycle kinetics in the alveolar epithelium. Am J Physiol 272: L1030-L1045, 1997.

14. Alcorn JF and Wright JR: Surfactant protein A inhibits alveolar macrophage cytokine production by CD14-independent pathway. Am J Physiol Lung Cell Mol Physiol 286: L129-L136, 2004.

15. Vazquez de Lara LG, Umstead TM, Davis SE and Phelps DS: Surfactant protein A increases matrix metalloproteinase-9 production by THP-1 cells. Am J Physiol Lung Cell Mol Physiol 285: L899-L906, 2003.

16. Hallman M, Maasilta P, Kivisaari L and Mattson K: Changes in surfactant in bronchoalveolar lavage fluid after hemithorax irradiation in patients with mesothelioma. Am Rev Respir Dis 141: 998-1005, 1990.

17. Sasaki R, Soejima T, Matsumoto A, Maruta T, Yamada K, Ota Y, Kawabe T, Nishimura H, Sakai E, Ejima Y, et al: Clinical significance of serum pulmonary surfactant proteins A and D for the early detection of radiation pneumonitis. Int J Radiat Oncol Biol Phys 50: 301-307, 2001.

18. Wynn TA: Cellular and molecular mechanisms of fibrosis. J Pathol 214: 199-210, 2008.

19. Wynn TA: Integrating mechanisms of pulmonary fibrosis. J Exp Med 208: 1339-1350, 2011.

20. Nagarajan D, Melo T, Deng Z, Almeida C and Zhao W: ERK/GSK3 $\beta /$ Snail signaling mediates radiation-induced alveolar epithelial-to-mesenchymal transition. Free Radic Biol Med 52: 983-992, 2012.

21. Verrecchia F and Mauviel A: Transforming growth factor-beta and fibrosis. World J Gastroenterol 13: 3056-3062, 2007.

22. Thannickal VJ, Toews GB, White ES, Lynch JP III and Martinez FJ: Mechanisms of pulmonary fibrosis. Annu Rev Med 55: 395-417, 2004.

23. Zhao L, Sheldon K, Chen M, Yin MS, Hayman JA, Kalemkerian GP, Arenberg D, Lyons SE, Curtis JL, Davis $\mathrm{M}$, et al: The predictive role of plasma TGF- $\beta 1$ during radiation therapy for radiation-induced lung toxicity deserves further study in patients with non-small cell lung cancer. Lung Cancer 59: 232-239, 2008.

24. Visse R and Nagase H: Matrix metalloproteinases and tissue inhibitors of metalloproteinases: Structure, function, and biochemistry. Circ Res 92: 827-839, 2003.

25. Atkinson JJ and Senior RM: Matrix metalloproteinase-9 in lung remodeling. Am J Respir Cell Mol Biol 28: 12-24, 2003.

26. López-Novoa JM and Nieto MA: Inflammation and EMT: An alliance towards organ fibrosis and cancer progression. EMBO Mol Med 1: 303-314, 2009.

27. Tan TK, Zheng G, Hsu TT, Wang Y, Lee VW, Tian X, Wang Y, Cao Q, Wang Y and Harris DC: Macrophage matrix metalloproteinase-9 mediates epithelial-mesenchymal transition in vitro in murine renal tubular cells. Am J Pathol 176: 1256-1270, 2010.

28. Cheng S and Lovett DH: Gelatinase A (MMP-2) is necessary and sufficient for renal tubular cell epithelial-mesenchymal transformation. Am J Pathol 162: 1937-1949, 2003.

29. Lee WH, Warrington JP, Sonntag WE and Lee YW: Irradiation alters MMP-2/TIMP-2 system and collagen type IV degradation in brain. Int J Radiat Oncol Biol Phys 82: 1559-1566, 2012. 
30. Strup-Perrot C, Vozenin-Brotons M-C, Vandamme M, Linard C and Mathé D: Expression of matrix metalloproteinases and tissue inhibitor metalloproteinases increases in X-irradiated rat ileum despite the disappearance of CD8a T cells. World J Gastroenterol 11: 6312-6321, 2005.

31. Yang K, Palm J, König J, Seeland U, Rosenkranz S, Feiden W, Rübe $\mathrm{C}$ and Rübe CE: Matrix-Metallo-Proteinases and their tissue inhibitors in radiation-induced lung injury. Int J Radiat Biol 83: 665-676, 2007.

32. Susskind H, Hymowitz MH, Lau YH, Atkins HL, Hurewitz AN, Valentine ES, Meek AG and Zucker S: Increased plasma levels of matrix metalloproteinase- 9 and tissue inhibitor of metalloproteinase-1 in lung and breast cancer are altered during chest radiotherapy. Int J Radiat Oncol Biol Phys 56: 1161-1169, 2003.

33. Daley WP, Peters SB and Larsen M: Extracellular matrix dynamics in development and regenerative medicine. J Cell Sci 121: 255-264, 2008
34. Shintani Y, Maeda M, Chaika N, Johnson KR and Wheelock MJ: Collagen I promotes epithelial-to-mesenchymal transition in lung cancer cells via transforming growth factor- $\beta$ signaling. Am J Respir Cell Mol Biol 38: 95-104, 2008.

35. Zeisberg M, Bonner G, Maeshima Y,et al: Renal fibrosis: Collagen composition and assembly regulates epithelial-mesenchymal transdifferentiation. Am J Pathol 159: 1313-1321, 2001.

36. Buckley ST, Medina C, Davies AM and Ehrhardt C: Cytoskeletal re-arrangement in TGF- $\beta 1$-induced alveolar epithelial-mesenchymal transition studied by atomic force microscopy and high-content analysis. Nanomedicine (Lond) 8: 355-364, 2012.

37. Kolb M, Margetts PJ, Sime PJ and Gauldie J: Proteoglycans decorin and biglycan differentially modulate TGF-beta-mediated fibrotic responses in the lung. Am J Physiol Lung Cell Mol Physiol 280: L1327-L1334, 2001.

38. Kasai H, Allen JT, Mason RM, Kamimura T and Zhang Z: TGF- $\beta 1$ induces human alveolar epithelial to mesenchymal cell transition (EMT). Respir Res 6: 56, 2005. 